\title{
From Reality to Virtuality: Re-discussing Cities with the Concept of the Metaverse
}

\begin{abstract}
Abidin Kemec*
Department of Office Services and Secretariat, Uşak University, Karahallı Vocational School, Uşak, Turkey.

*Correspondence: abidinkemec@gmail.com (Abidin Kemec, PhD, Department of Office Services and Secretariat, Uşak University, Karahallı Vocational School, Uşak, Turkey).
\end{abstract}

\begin{abstract}
The increase in the transaction volume of cryptocurrencies, the widespread use of blockchain technology, and increase in the number of augmented reality devices (virtual, augmented, and mixed reality) have led to the formation of a suitable environment for Metaverse. Metaverse is a virtual or augmented reality created beyond the physical world via an internet connection with augmented reality devices, game consoles, computers, tablets or mobile phones. This universe may be beyond the real world, or it is created by the virtualization of real world objects to a certain extent. While Metaverse has the opportunity to be applied comfortably in all areas of daily life, it can also be designed as an urban policy design tool. In the study, the conceptual framework of the metaverse will be presented, the metaverse investments of global companies will be determined, the concept of metaverse will be examined in the context of virtual reality relationship, the reflections of metaverse applications on the city will be examined, and finally, the effects and role of the metaverse in urban policy production will be discussed and conclusions and evaluations will be given.
\end{abstract}

Keywords: Metaverse, City reality, Virtuality, Reality, Augmented Reality, and Metaverse applications.

\section{INTRODUCTION:}

The word 'metaverse' was first used by Neal Stephenson. He described it as a gigantic virtual universe parallel to the world, from the metaverse in the dystopian and cyberpunk novel Snow Crash (Joshua, 2017). Since then, Facebook CEO Mark Zuckerberg has had a significant impact on the concept of the metaverse. Ever since Zuckerberg announced his decision to rebrand the company as Meta on October 28,2021 , there has been intense interest in the concept of the "metaverse". In addition to Zuckerberg's statement, developing information and communication technologies, mobile phone applications and social media platforms also contributed significantly.

As the number of people using the Internet increased, cyberspace began to develop. Virtual universes have been created through devices in various fields, including social media applications, videos, meeUniversePG I www.universepg.com tings and games. Virtual universes, although permanent and disconnected, have brought different levels of digital transformation to users. The term "Metaverse" has facilitated digital transformation in all areas of life. At the heart of metadata is an immersive vision of the internet as a huge, joint, shared and permanent space. Driven by technologies such as augmented reality, blockchain, and artificial intelligence (AI), the metaverse concept big bang is imminent (Lee et al., 2021).

In virtual spaces created in the metaverse, people can connect with each other through digital avatars. The digital avatar can act as a close replica of a real person. People can create their digital avatars in virtual reality. This technology could allow coworkers to sit and chat over coffee, escape worries by attending virtual concerts, and stay in a virtual space that feels real (Analytics steps, 2021). 


\section{Metaverse Concept}

On a global scale, an estimated every ten years, technology platforms have undergone a paradigm shift. Computer communication in the 1990s, in order of paradigms in the following decades; website, mobile communication and the "metaverse. In the Metaverse concept, digital users/avatars interact with each other socially and economically through 3D virtual and multi-user online environments. Now the real space is connected to the virtual space through metastore transformations (Lee, 2021).

Looking at the history of the metaverse, the greatest progress has been made from the video game industry through games such as Wolfenstein 3D, Duke Nukem 3D, Ever-Quest, Ultima Online and Doom. In these games, 3D game playing experience is provided for the users (Cammack, 2010). Metaverse is an advanced form of online gaming. In terms of the connection between reality and virtuality, the metaverse has a stronger connection between reality and virtuality. The way Metaverse users participate is also different from before. Users can participate more freely than in existing online games, with the way they create their own stories rather than narratives such as role-playing and drama using the space (Han et al., 2021). Caulfield defines the concept of metaverse as synergistic, jointly and immersive shared three-dimensional virtual world(s) (Caulfield, 2021). Metaverseis virtual shared spaces that create a universe, allowing the user to choose an avatar, build homes and businesses, hold meetings, attend classes, find partners, and do the seemingly impossible (Srushti, 2021).

In another study, it was emphasized that the metaverse serves as a virtual spatial background that enables users to participate in social, economic and cultural activities through avatars (Hendaoui et al., 2008). I can be used to incorporate intelligence into the Metaverse for enhanced user experience such as efficient object creation, intelligent chatbots, and user-generated content. For example, Epic Games company's Meta Human project used AI to quickly create lifelike digital characters (Lim et al., 2022). For a metaverse to meet real-world requirements, at least; a shared 3D environment, avatar, communication (interpersonal, group, audience), real-time interaction, creative expression (personal and environmental) and financial resources are needed (Cammack, 2010). There with all the metaverse is a com- plex digital environment based on seven different layers. These layers (xrtoday.com, 2021a):

1) Infrastructure- 5G, Wi-Fi and processors

2) Human interface - devices used to connect in metadata (VR headsets, AR glasses, haptics, and other technologies)

3) Decentralization- Blockchain, edge computing, artificial intelligence

4) Spatial computing- 3D visualization and modeling frameworks

5) Creative economy- Various design tools, digital assets and e-commerce establishments

6) Discovery- Ads, social media, ratings, reviews, etc. A content engine that provides inter-action, including.

7) Experiences- Game, event, work, shopping etc. for digital applications.

Metaverse is a new investment area for companies. The world's leading global companies carry out R\&D studies for different kind of software and hardware. The goals of the companies on the metaverse Nare shown in the Table 1. Apple virtual reality equipment; Creating content in the Amazon media industry; Creating a virtual city in Decentraland; Google metadata transfer; Facebook invests in providing unique experiences on social networks.

\section{Metaverse, Reality-Virtual Relation:}

Virtual, augmented, augmented and mixed reality applications are the leading technologies that are effective in the use of Metaverse in many areas today. These reality practices are linked with many technological developments in the fields of entertainment, health, education, automotive industry (Ling et al., 2020; Zhou et al., 2020; Lawson et al., 2016; Adikari et al., 2020; Huang et al., 2020; Cammack, 2010).

While in earlier times the use of XR was limited to "in the laboratory" or only in private areas, it has now led to wider acceptance in society, such as in civil defense, aviation, emergency preparedness and evacuation planning, and almost all educational disciplines, besides the private sector (Çöltekin et al., 2020). Whether on devices in virtual and augmented reality or on a display or console, the promise of the metaverse is to allow a greater overlap of virtual and real lives in wealth, socializing, productivity, shopping, and entertainment (Clark, 2021). 
Table 1: Companies Targeting Metaverse Investment (Analyticssteps, 2021; Xrtoday, 2021a).

\begin{tabular}{|c|c|}
\hline Companies & Goals \\
\hline NIVDIA & $\begin{array}{l}\text { Build a real-time, physically accurate simulation built for creators, designers, engineers, } \\
\text { and researchers who need to collaborate in a virtual space. }\end{array}$ \\
\hline Apple & Producing VR equipment. \\
\hline Unity Software & $\begin{array}{l}\text { Unity has the ability to provide artists and creators with the tools to succeed in the } \\
\text { metaverse, so anyone can work together to create content for the metaverse. }\end{array}$ \\
\hline Roblox & $\begin{array}{l}\text { It is planned to design a metaverse that meets the needs of players and empowers its } \\
\text { community with tools that facilitate a safe environment and help maintain virtual } \\
\text { relationships. Spatial voice chat will enable people to voice chat in virtual environment. }\end{array}$ \\
\hline Epic & $\begin{array}{l}\text { Setting the stage for social experiences such as virtual music concerts and dance parties, } \\
\text { where people can dress up as their avatars and build islands. }\end{array}$ \\
\hline Autodesk & Creating virtual worlds for play and entertainment. \\
\hline Microsoft & $\begin{array}{l}\text { Creating mixed reality with avatars that allows people to project themselves directly onto } \\
\text { others in the VR and AR worlds. }\end{array}$ \\
\hline Amazon & To create a metadata warehouse in the media industry. \\
\hline Google & To provide metadata transfer. \\
\hline Facebook & $\begin{array}{l}\text { Enable VR Messaging and create a place where buyers, sellers and creators can } \\
\text { exchange digital goods. }\end{array}$ \\
\hline Decentraland & Building a real estate market in the VR world. \\
\hline
\end{tabular}

\section{Chapter 1}

\section{Extended Reality}

Augmented reality refers to all real and virtual combined environments and human-machine interactions created by computer technology, wearable technology, and game consoles. The concept of augmented reality encompasses all forms of reality such as augmented reality, virtual reality, mixed reality. In addition, augmented reality can be defined as a simple concept that combines three realities in a single term (Medium, 2018). XR encompasses immersive technologies that "expand" experienced reality by involving users in a human-machine converged reality through an integrated cyber and physical environment using computers and wearable's (Alnagrat et al., 2021).

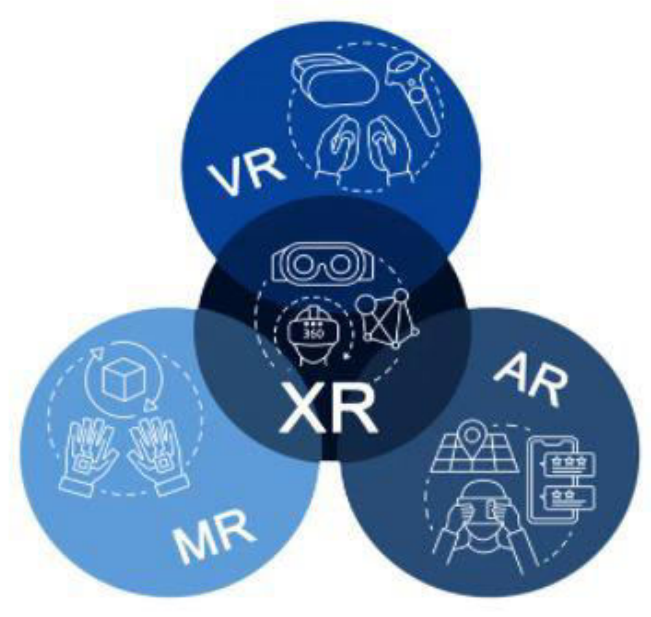

Fig. 1: Applications of Extended Reality (envisionis.com, 2022).
Technologies seen as tools to create unique environments, experiences and interactions, XR represents a powerful opportunity for businesses and consumers alike. The XR market, valued at US\$26.05 billion in 2020, has grown at an incredible pace. Experts think that XR will reach a value of $\$ 463.7$ billion by 2026 and achieve a growth of over $62.67 \%$. The market of augmented reality applications has seen a significant increase in 2020 and 2021.

The pandemic (COVID-19) has increased interest in the creation of virtual experiences in many industries to strengthen business and collaboration. XR has also developed into institutionalism. Nowadays companies now include augmented reality when planning their digital transformation strategies (Xrtoday, 2021b). In the research report published by the Accenture Company in 2021, it was claimed that global companies are heavily transitioning to augmented reality technology. While companies take initiatives to increase employee participation and provide more effective and flexible learning opportunities, they assign important missions to $\mathrm{XR}$ technologies. In addition, a highly effective learning experience can be experienced by creating a safe and fully interactive environment (Accenture, 2021).

Extended reality glasses can be made more functional by adding different equipment. In order for these glasses to serve various purposes; Camera, microphones, speaker and sensors can be mounted. 


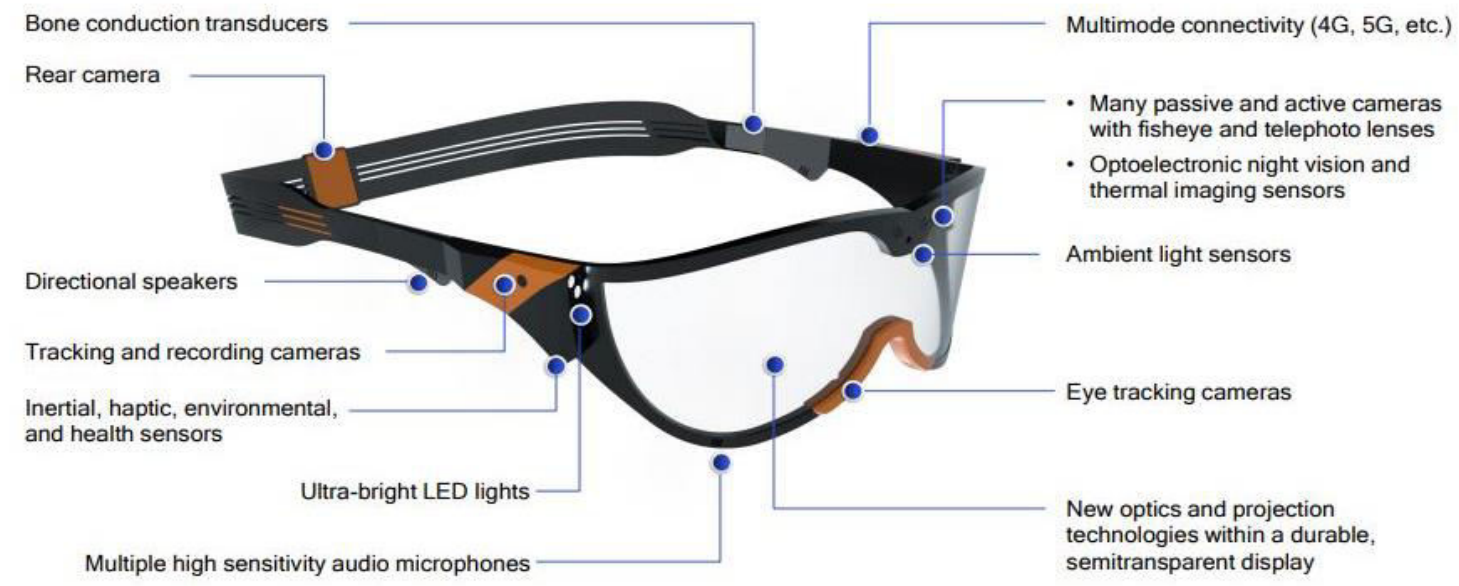

Fig. 2: XR Glasses (Cualcomm, 2020).

\section{Chapter 2}

\section{Augmented Reality}

Augmented reality is the realization of virtual environment by adding virtual information to a physical real world environment. Augmented reality adds virtual information not only to the real environment, but also to video and game streams, giving the user a simpler view. Augmented reality is a visual augmentation technique that transfers computergenerated data (text, video, graphics, and GPS data) onto a real-world image as captured from the camera of a tablet, notebook, computer, mobile phone, or game console (Kounavis, 2012). Augmented reality applications; fixed indoor, fixed outdoor, mobile indoor, mobile outdoor and mobile indoors and outdoor systems are divided into five categories. Mobile systems allow the user to move around without being limited to a room, thus making it easy for the user to move around using a wireless system. Fixed systems cannot be moved. In this system, users can only use the system where it is installed (Carmigniani et al., 2011).

\section{Chapter 3}

\section{Virtual Reality}

Virtual reality is a human-computer desire simulating an advanced world. They can browse virtually online (Zheng et al., 1998). VR is an artificial application created by different devices in a real-life environment. This application helps users experience the transformed reality in a different way by adapting the senses such as sight and hearing in addition. Virtual reality has features such as disconnecting experiences and interactions from the real world or blending them with the real world, making real and UniversePG I www.universepg.com virtual similar. For these reasons, the number of users has increased rapidly in recent years (Ramos et $a l ., 2018)$. The main aim of the Virtual Reality system is to use technology to change reality and create an immersive environment. It means that the display must be completely immersive, with a wide field of view and 3D graphics rendered as realistically as possible. The land of view is the size of the user's screen. So the larger the land of view, the higher the intensity level (Azuma, 1997).

\section{Chapter 4}

\section{Mixed Reality}

Ideas have been put forward that AR and VR are closely related to each other and that it is necessary to deal with the two concepts together. In augmented reality (AR), the real world is enriched with virtual content. In augmented virtuality (AV), most of the content is virtual, but the objects found in the world have been added to some extent. In mixed reality (MR), objects that exist in the real and virtual worlds are blended (Skarbez et al., 2021). In a study published in 1994 by Paul Milgram and Fumio Kishino, mixed reality was able to mimic properties of physical reality, creating a world in which the participant observer is completely immersed in a virtual world, where the physical laws governing gravity, time, and material properties of the existing or fictional realworld environment no longer apply but it is also described as a view that can cross borders. A real environment is any environment consisting only of real objects (Fig. 2). A virtual environment is an environment made up of virtual objects. In this framework, a general Mixed Reality (MR) environment, real world and virtual world objects are presented on a single screen. 


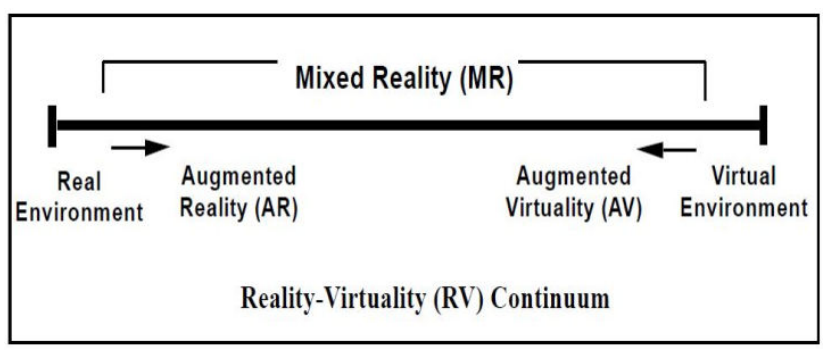

Fig. 3: Reality and Virtuality Plane (Milgram et al., 1995).

When comparing the three realities (Fig. 3), augmented reality and mixed reality headsets are used, while special headsets or smart glasses are used in virtual reality. While creating a completely digital environment in virtual reality, it is presented in augmented and mixed reality blended with real life. The main difference between augmented reality and mixed reality relates to the level of awareness of virtual objects. In mixed reality, there are perfectly rendered virtual objects that are indistinguishable from real objects, while in augmented reality virtual objects can be described according to their nature and behavior.

Table 2: Collation of the Reality Fields (Rokhsaritalemi et al., 2020)

\begin{tabular}{|c|c|c|c|}
\hline Features & Augmented Reality & Virtual Reality & Mixed Reality \\
\hline Display device & Headsets optional & $\begin{array}{c}\text { Mostly using Special } \\
\text { headset or smart glasses }\end{array}$ & Headsets optional \\
\hline Image sounce & $\begin{array}{l}\text { Combination of } \\
\text { computer-generated images } \\
\text { and real-life objects }\end{array}$ & $\begin{array}{l}\text { Computer graphics or } \\
\text { real images produced by } \\
\text { a computer }\end{array}$ & $\begin{array}{l}\text { Combination of } \\
\text { computer-generated images } \\
\text { and real-life objects }\end{array}$ \\
\hline Environment & $\begin{array}{l}\text { Both virtual and real-life } \\
\text { objects are seamlessly blended }\end{array}$ & Fully digital & $\begin{array}{c}\text { Both virtual and real-life } \\
\text { objects are } \\
\text { seamlessly blended }\end{array}$ \\
\hline Perspective & $\begin{array}{c}\text { Virtual objects behave based } \\
\text { on user's perspective in the } \\
\text { real world }\end{array}$ & $\begin{array}{c}\text { Virtual objects will } \\
\text { change their position } \\
\text { and size according to the } \\
\text { user's perspective in the } \\
\text { virtual world }\end{array}$ & $\begin{array}{c}\text { Virtual objects behave based } \\
\text { on user's perspective in the } \\
\text { real world }\end{array}$ \\
\hline Presence & $\begin{array}{l}\text { Feeling of still being in the real } \\
\text { world, but with new elements } \\
\text { and objects superimposed }\end{array}$ & $\begin{array}{l}\text { Feeling of being } \\
\text { transported somewhere } \\
\text { else with no sense of the } \\
\text { real world }\end{array}$ & $\begin{array}{l}\text { Feeling of still being in the } \\
\text { real world, but with new } \\
\text { elements and objects } \\
\text { superimposed }\end{array}$ \\
\hline Awareness & $\begin{array}{l}\text { Virtual objects can be } \\
\text { identified based on their } \\
\text { nature and behavior, such as } \\
\text { floating text that follows a user }\end{array}$ & $\begin{array}{l}\text { Perfectly rendered } \\
\text { virtual objects that } \\
\text { cannot be distinguished } \\
\text { from real objects }\end{array}$ & $\begin{array}{l}\text { Perfectly rendered virtual } \\
\text { objects that cannot be } \\
\text { distinguished from } \\
\text { real objects }\end{array}$ \\
\hline
\end{tabular}

\section{Different application Areas Of Metaverse In The city}

The application of metaverse finds its practical application in many areas (science, health, culture, art, economy and daily life) of the city. In the report published by Thomas Alsop, among the smart cities best XR/AR/VR/MR applications in America in 2020, navigation solutions and improvements in urban planning are the two best applications of innovator technologies in smart cities. These two are respectively; smart traffic signaling management, smart parking and smart building management systems follow (statista.com, 2020). Realtors use XR allowing buyers to view and experience properties without being physically present. Interior designers and architects also experiment with their designs and can avoid mistakes. The comfort of seeing a whole house while living in a another city or even in a different country and seeing the harmony of the furniture with the interior design of the house will be a great breakthrough for the real estate sector (logicsimplified. com, 2022). As an example, IKEA's latest mobile app, IKEA Place, uses XR to make a profound impact on the way we shop for furniture at home. Those who want to shop through the mobile application can choose a product from the catalog and place digital furniture anywhere in the room. The selected product is quickly fitted to the specified area (viget, 2017).

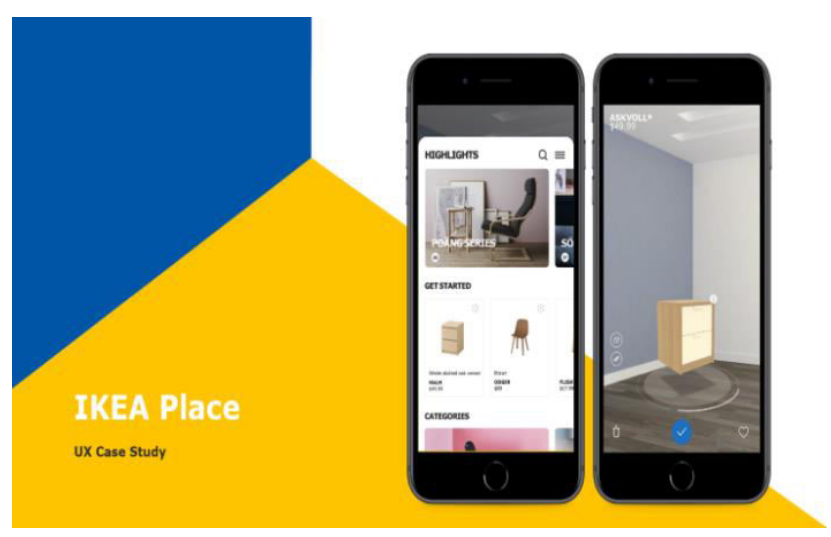

Image 1: IKEA Place Mobil Application (ikea.com, 2022). 
Metaverse offers a completely different experience for customers/users to co-create experiences in the retail industry and to better design services, increase customer engagement, and strengthen communication. The product types offered by Metaverse retailing span a range from virtual digital to digital for offline to purchasing real-life physical items for use (Gadalla et al., 2013). There is a direct link between the recent increase in NFT (Non-Fungible Token) sales and Metaverse. Many artifacts sold as NFTs are protected in the digital worlds. These works will be exhibited in the Metaverse universes in the future and will be sold by auction. In the Metaverse universe, these digital artworks are put up for sale by signing contracts and transferring money securely, thanks to blockchain technology. Through blockchain-based smart contract mechanisms, metaverse service providers can manage and automate complex interactions between various entities in the Metaverse, such as users and digital content creators (Nguyen et al., 2021). Recently, it is on the agenda to get a collection of virtual terrain, marketplace, avatar or NFT in different metaverse universes. Within the scope of the metaverse, two different universes are created, either a copy of the real world or independent of the world. Decentraland, the first fully decentralized virtual world, is controlled by computer codes through the Decentralized Autonomous Organization, which owns smart contracts and assets (decentraland.org, 2022). Next Earth is the only blockchain-based virtual land ownership platform on a full copy of the world. On the Next Earth platform, one can instantly buy and sell digital land and land NFT collections in a non-intermediary, NFT-based, transparent manner. Next Earth has three land types: water, urban and non-urban (nextearth.io, 2022). Decentraland Company has implemented Metaverse in real estate by combining virtual reality and blockchain technology. Decentraland has made it possible to buy land, a virtual real estate, using the cryptocurrency MANA. Users can freely place buildings on land purchased from Decentraland, generate income by adding billboards to buildings, or hold exhibitions by collecting rare digital content. These unique assets are made through smart contracts that enable decentralized autonomous organization, transactions regarding stocks, bonds, loans, mortgages, smart property (Jeon et al., 2021). Samsung plans to enter the metadata warehouse by opening a store in Decentraland (MANA).
The company will open the shop for a limited time to offer users unusual digital adventures. Named Samsung $837 \mathrm{X}$ in reference to the physical store located at 837 Washington Street, it will still be open for a limited time. The purpose of this store is to offer digital adventures to its users. Users will be able to join a game to get NFT and wearable's. It has also been noted that these NFTs will be available in limited quantities (medium.com, 2022).

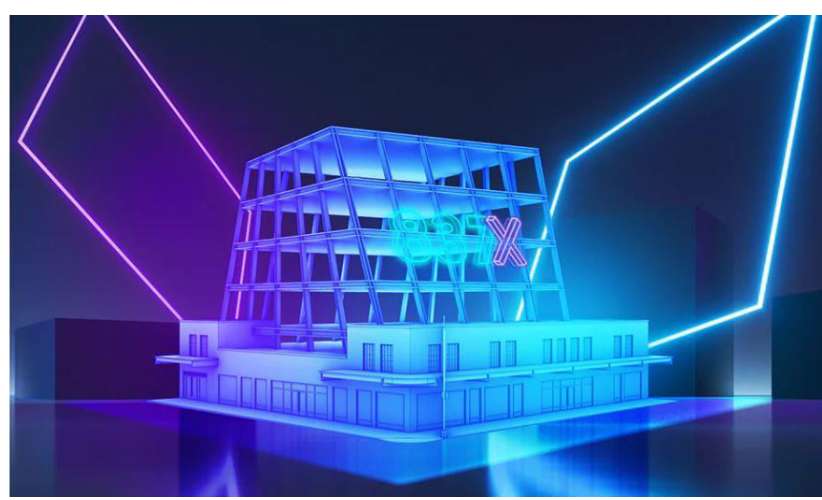

Image 2: Samsung Company's Metaverse Store (samsung.com, 2022)

Another dimension of Metaverse is that it offers a digital economy environment where users can create, buy and sell goods. Cryptocurrencies can be used in the metaverse universe. Cryptocurrency exchanges list many zcoins in relation to the metaverse. Metaverse coins are (coinmarketcap.com, 2022); Decentraland (MANA), Axie Infinity (AXS), The Sandbox (SAND), THETA (THETA), UFO Gaming (UFO), Enjin Coin (ENJ), WEMIX (WEMIX), Starlink (STARL). These platforms act as virtual reality platforms that allow users to create content and applications, create, experience and monetize digital assets in the form of a game, interacting without violating personal data, powered by the Ethereumblockchain.

\section{CONCLUSION:}

There has been a general rise in digital literacy levels with the increase in internet infrastructure and usage, the number of people using technological devices and digital applications. As the level of digital literacy increases, the journey from physical to virtual has become more reliable, understandable and usable. Innovative applications such as the metaverse of the digital age have been met by millions of people. Firms engaged in physical merchandising activities (Samsung, Zara, Ikea etc.) have opened virtual stores. Game companies have provided a stronger connection between reality and virtuality in 
online games, allowing players to move more freely. Thanks to the metaverse applications of the pandemic in business, shopping, education, sports, social, cultural and artistic activities in cities, a new experience has been experienced. In parallel with the increase in the internet infrastructure and usage, the number of people using technological devices and digital applications, there has been a general increase in people's digital literacy levels. As the level of digital literacy increases, the journey from physical to virtual has become more reliable, understandable and usable. Firms engaged in physical merchantdising activities (Samsung, Zara, Ikea, etc.) have provided users with a unique experience with the virtual stores they opened in different metaverse universes. Game companies have provided a stronger connection between reality and virtuality in online games, allowing players to move more freely. Due to the pandemic, participation in cultural and artistic activities where people participate collectively has decreased significantly all over the world. With Metaverse applications, a new experience has been experienced in business, shopping, education, sports, social, cultural and artistic activities in cities. Metaverse applications have radically transformed property relations. It has been possible to purchase and lease real estate from different metaverse universes. Establishing a world-independent virtual universe (Decentraland) and real-world lands and NFTs can be rented or purchased again virtually (Next Earth) over the metaverse. Metaverse applications are seen as a blessing of the new age. However, as with all digital applications, it is also concerned that it will pose a risk due to personal privacy and data security.

\section{ACKNOWLEDGEMENT:}

I would like to thank the authors of the literature sources I have used in this article.

\section{CONFLICTS OF INTEREST:}

The author of the article has no conflict of interest with any institution or person.

\section{REFERENCES:}

1) Accenture, (2021). The mobile future of extended reality (xr), https://www.accenture.com/_acnmedia/PDF-164 /Accenture-Immersive-learning.pdf\#zoom $=40$

2) Adikari, S. B., Ganegoda, N. C., Meegama, R. G., Wanniarachchi, I. L. (2020). Applicability of a single depth sensor in real-Time 3D clothes simulation: Augmented reality virtual UniversePG I www.universepg.com dressing room using kinect sensor. Advances in Human-Computer time Interaction, 2020.

3) Alnagrat, A. J. A., Ismail, R. C., \& Idrus, S. Z. S. (2021). Extended reality (xr) in virtual laboratories: A review of challenges and future training directions. In Journal of Physics: Conference Series, IOP Publishing, 1874(1).

4) Analyticssteps.com, (2021). 8 top companies leading the metaverse technology, https://www.analyticssteps.com/blogs/8-top-com panies-leading-metaverse-technology

5) Azuma, R. T. (1997). A survey of augmented reality. Presence: Teleoperators \& Virtual Environments, 6(4), 355-385.

6) Binance. (2021). 6 global companies building up the metaverse,

https://academy.binance.com/en/articles/globalcompanies-building-up-the-metaverse

7) Cammack, R. G. (2010). Location-based service use: a metaverse investigation. Journal of Location Based Services, 4(1), 53-65.

8) Carmigniani, J., Furht, B., Anisetti, M., Ceravolo, P., Damiani, E., Ivkovic, M. (2011). Augmented reality technologies, systems and applications. Multimedia tools and applications, 51(1), 341-377.

9) Clark, P.A. (2021). The metaverse has already arrived. Here's what that actually means, https://time.com/6116826/what-is-the-metaverse/

10) Caulfield, B. (2021). "What is the metaverse?" The official Nvidia Blog, https://blogs.nvidia.com/blog/2021/08/10/whatis-the-metaverse/

11) Coinmarketcap.com, (2022), Largest Metaverse by market cap, https://coinmarketcap.com/tr/view/metaverse/

12) Çöltekin, A., Lochhead, I., Madden, M., et al., (2020). Extended reality in spatial sciences: A review of research challenges and future directions. ISPRS International Journal of GeoInformation, 9(7), 439.

13) Decentraland.org, (2022). Decentraland, https://decentraland.org/

14) Envision, (2022). Envision innovative solutions to animate U.S. Capitol using augmented reality, https://envision-is.com/xr/

15) Gadalla, E., Keeling, K., \& Abosag, I. (2013). Metaverse-retail service quality: A future framework for retail service quality in the 3D internet. Journal of Marketing Management, 29(13-14), 1493-1517. 
16) Han, J., Heo, J., You, E. (2021). Analysis of metaverse platform as a new play culture: Focusing on roblox and ZEPETO. In Proceedings of the $2^{\text {nd }}$ International Conference on Human-centered Artificial Intelligence (Computing4 Human 2021). CEUR Workshop Proceedings, Da Nang, Vietnam.

17) Hendaoui, A., Limayem, M., Thompson, C.W. (2008). 3D social virtual worlds: research issues and challenges. IEEE Internet Compu-ting, 12(1), 88 92, https://doi.org/10.1109/MIC.2008.1

18) Huang, H., Lin, C., Cai, D. (2020). Enhancing the learning effect of virtual reality 3D modeling: a new model of learner's design collaboration and a comparison of its field system usability. Universal Access in the Information Society, 1-12.

19) Ikea.com. (2022). IKEA Place,

https://about.ikea.com/en/newsroom/2018/03/19/ ikea-place-app-launches-on-android-allowing-mi llions-of-people-to-reimagine-home-furnishingsusing-ar?assetID=B724AB72-104C-4703-B941D617E402836A

20) Jeon, H. J., Youn, H. C., Ko, S. M., \& Kim, T. H. (2021). Blockchain and AI meet in the metaverse,

https://www.intechopen.com/chapters/77823

21) Joshua, J. (2017). Information bodies: Computational anxiety in Neal Stephenson's Snow Crash. Interdisciplinary Literary Studies, 19 (1), 17-47.

22) Kounavis, C. D., Kasimati, A. E., \& Zamani, E. D. (2012). Enhancing the tourism experience through mobile augmented reality: Challenges and prospects. International Journal of Engineering Business Management, 4, 10.

23) Lawson, G., Salanitri, D., \& Waterfield, B. (2016). Future directions for the development of virtual reality within an automotive manufacturer. Applied ergonomics, 53, 323-330.

24) Lee, L. H., Braud, T., Zhou, P., Wang, L., P. (2021). All one needs to know about metaverse: A complete survey on technological singularity, virtual ecosystem, and research agenda. arXiv preprint.

https://doi.org/arXiv:2110.05352

25) Lee, J. Y. (2021). A study on metaverse hype for sustainable growth. International journal of advanced smart convergence, 10(3), 72-80.
26) Liang, C. J., Start, C., Boley, H., et al., (2020). Enhancing stroke assessment simulation experience in clinical training using augmented reality. Virtual Reality, 1-10.

27) Lim, W. Y. B., Xiong, Z., Niyato, D., et al., (2022). Realizing the metaverse with edge intelligence: A match made in heaven. arXiv preprint. https://doi.org/arXiv:2201.01634

28) Logicsimplified.com, (2022). Extended Reality (XR): The rising star of virtual world, https://logicsimplified.com/newgames/extendedreality-xr-the-rising-star-of-virtual-world/

29) Medium. (2018). What really is the difference between AR/MR/VR/XR ?

https://medium.com/@northof41/what-really-is-t he-difference-between-ar-mr-vr-xr-35bed1da1a4e

30) Medium.com, (2022). Samsung enters the metaverse by opening a store in Decentraland (MANA),

https://medium.com/coinmonks/samsung-entersthe-metaverse-by-opening-a-store-in-decentralan d-mana-11a4e619d7d5

31) Milgram, P., Takemura, H., Utsumi, A., Kishino, F. (1995, December). Augmented reality: A class of displays on the reality-virtuality continuum. In Tele manipulator and telepresence technologies, 2351, 282-292.

32) Nextearth.io, (2022). Next Earth, https://nextearth.io/

33) Nguyen, C. T., Hoang, D. T., Nguyen, D. N., Dutkiewicz, E. (2021). MetaChain: A novel blockchain-based framework for metaverse applications. arXiv preprint. https://doi.org/arXiv:2201.00759

34) Qualcomm, (2020). The mobile future of extended reality (xr), Qualcomm Technologies, Inc.,

https://www.qualcomm.com/media/documents/fi les/the-mobile-future-of-extended-reality-xr.pdf

35) Ramos, F., Trilles, S., Torres-Sospedra, J., Perales, F. (2018). New trends in using augmented reality apps for smart city contexts. ISPRS International Journal of Geo-Information, 7(12), 1-23.

36) Rokhsaritalemi, S., Sadeghi-Niaraki, A., Choi, S. M. (2020). A review on mixed reality: Current trends, challenges and prospects. Applied Sciences, 10(2), 636.

37) Sami HM. (2021). Portfolio construction using financial ratio indicators and classification 
through machine learning, Int. J. Manag. Account. 3(4), 83-90. https://doi.org/10.34104/ijma.021.083090

38) Skarbez, R., Smith, M., Whitton, M. C. (2021). Revisiting Milgram and Kishino's reality-virtuality continuum. Frontiers in Virtual Reality, 2, 27.

39) Statista.com. (2020). XR/AR/VR/MR applications in the United States in 2020, https://www.statista.com/statistics/1185244/appli cations-immersive-technologies-xr-ar-vr-mr-sma rt-cities/

40) Samsung.com, (2022). Samsung 837X, https://www.samsung.com/us/explore/metaverse$837 \mathrm{x} /$

41) Srushtiimx.com, (2021). Does the virtual world advertising work in metaverse, https://srushtiimx.com/blog/virtual-world-adverti sing-work-in-metaverse/
42) Viget, (2017). A definitive guide to navigating the landscape of extended reality, https://www.viget.com/articles/xr-vr-ar-mr-what s-the-difference/

43) Xrtoday.com, (2021a). What is extended reality? Demystifying XR tech, https://www.xrtoday.com/mixed-reality/what-is-e xtended-reality/

44) Xrtoday.com, (2021b). Who is building the metaverse? A group of $160+$ companies https://www.xrtoday.com/virtual-reality/who-is-b uilding-the-metaverse-a-group-of-160-companiesand-you/

45) Zheng, J. M., Chan, K. W., Gibson, I. (1998). Virtual reality. IEEE Potentials, 17(2), 20-23.

46) Zhou, X., Tang, L., Lin, D., Han, W. (2020). Virtual \& augmented reality for biological microscope in experiment education. Virtual Reality \& Intelligent Hardware, 2(4), 316-329.

Citation: Kemec A. (2022). From reality to virtuality: re-discussing cities with the concept of the metaverse, Int. J. Manag. Account. 4(1), 12-20. https://doi.org/10.34104/ijma.022.00120020 @) () 